\title{
Medical Image Enhancement by using Cellular Neural Networks
}

\author{
A Gacsádi, C Grava, A Grava \\ University of Oradea, Romania
}

\begin{abstract}
The paper presents a medical image enhancement method taking the noise reduction and the contrast enhancement into consideration, as well as the possibility of implementation on an existing cellular neural network universal chip (CNN-UC), in a single step, by using only linear templates of $3 \times 3$ dimensions. Due to complete parallel processing, computing-time reduction is achieved.
\end{abstract}

\section{Introduction}

The problem of medical image enhancement, became more and more important in the last decade. Another challenging in medical imaging is to obtain real-time applications. In this paper we propose a fully-parallel solution to achieve the medical image enhancement.

The method, based on Cellular Neural Networks (CNN) [1], is suitable for real-time processing.

Cellular Neural Networks (CNN) [1], CNN-Universal Machine's (CNN-UM) [2] architecture and CNN Universal-Chip (CNN-UC) [3] has proved to be a competing alternative to classical computational techniques. A Cellular Neural Network is a 2-dimensional rectangular structure, composed by identical analogical non-linear processors, named cells [2]. The CNN allows fully-parallel image processing, a given processing being executed simultaneously for the entire image.

The state equation of a cell is [1]:

$\dot{x}=\frac{d x_{i j}}{d t}=-x_{i j}+\sum_{C_{l k} \in N_{r}} A_{i j, l k} \cdot y_{l k}+\sum_{C_{l k} \in N_{r}} B_{i j, l k} \cdot u_{l k}+z_{i j}(1)$

where $x_{i j}$ represent the state, $u_{i j}$ is the input, $y_{i j}$ is the output and $z_{i j}$ is the threshold of the cell $(i, j) . A_{i j, k l}$ is the feed-back operator and $B_{i j, k l}$ is the input operator. The ensemble $(A, B, z)$ is named template. $C_{l k}$ are the cells from a $r$-order neighborhood $N_{r}$ of the cell $(i, j)$ :

$$
N_{r}(i, j)=\{C(l, k) \mid \max \{|l-i|,|k-j|\} \leq r\}
$$

with $1 \leq i, l \leq L, 1 \leq j, k \leq K, r \leq \min (L, K) . K$ and $L$ are the dimensions of the network.

The structure of a cell $C_{i j}$ is presented in Figure 1:

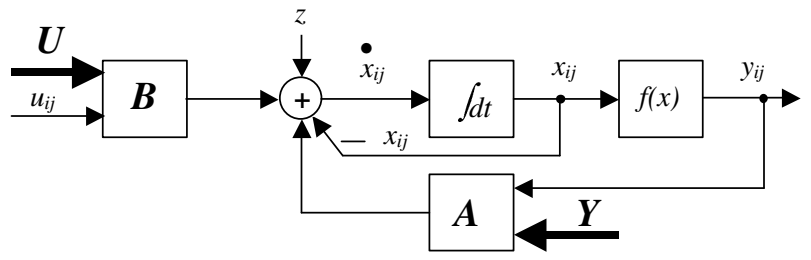

Figure 1. System structure of a cell $C_{i j}$.

In Figure 2, the signal flow structure of a CNN with a $3 \times 3$ neighborhood [2].

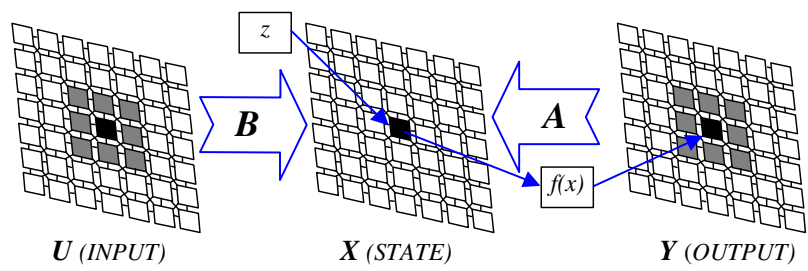

Figure 2. Signal flow structure of a $\mathrm{CNN}$ with a $3 \times 3$ neighborhood.

\section{PDE image processing}

Generally, the image processing tasks could be described by a PDE (Partial Differential Equation). Whatever the image processing described by a PDE is, a considerable computing power is necessary. Regarding this problem, the Cellular Neural Networks (CNN [1]) proved to be very helpful regarding the real-time image processing, as well as solving some partial differential equations [4,5]. Reduction in computing time due to parallel processing, can be obtained only if the processing algorithm can be implemented on a Cellular Neural Networks-Universal Chip (CNN-UC [3]), having the architecture of a Cellular Neural Networks-Universal Machine (CNN-UM [2]).

Recently, the interest in using partial differential equations (PDE-s) has grown in the field of image processing and analysis $[6,10]$. Let us consider a grayscale image $\Phi_{0}(\mathrm{x}, \mathrm{y}), \Phi_{0}: \mathbf{R}^{2} \rightarrow \mathbf{R}$. The processing of this image, according to an algorithm based on an operator, can be described by the following partial differential equation: 


$$
\frac{\partial \Phi}{\partial \mathrm{t}}=\mathrm{F}[\Phi(\mathrm{x}, \mathrm{y}, \mathrm{t})]
$$

where an artificial parameter $\mathrm{t}$ has been used and $F$ is the operator which characterizes the desired processing algorithm $\left(F: \mathbf{R}^{2} \rightarrow \mathbf{R}\right)$. In general, function $F$ depends on the initial image, and its first and second order spatial derivatives. The final image is obtained from processing as a solution of this partial differential equation.

The partial differential equation can be obtained from variational problems. The image results from the minimization of a cost function:

$$
\arg \left\{\operatorname{Min}_{\Phi} \mathrm{E}(\Phi)\right\},
$$

where $E$ is a given energy function. Minimizing $E, \Phi$ can be obtained from the condition: $F(\Phi)=0$, which results in a steady state solution of the equation:

$$
\frac{\partial \Phi}{\partial \mathrm{t}}=\mathrm{F}(\Phi)
$$

where $t$ is also an artificially introduced parameter.

The PDE formulation of image processing gives us the possibility of combining the algorithms. If, e.g. two distinct processing are given by the equations:

$$
\frac{\partial \Phi}{\partial \mathrm{t}}=\mathrm{F}_{1}[\Phi(\mathrm{x}, \mathrm{y}, \mathrm{t})] \text { and } \frac{\partial \Phi}{\partial \mathrm{t}}=\mathrm{F}_{2}[\Phi(\mathrm{x}, \mathrm{y}, \mathrm{t})] \text {, }
$$

a complex image processing is resulted by the combination of these equations as follows:

$$
\frac{\partial \Phi}{\partial \mathrm{t}}=\mathrm{F}_{1}+\lambda \mathrm{F}_{2}
$$

where $\lambda \in \mathbf{R}^{+}$. The differential equation (7) can be obtained from the minimization of energy $E_{1}+\lambda E_{2}$, if $F_{1}$ and $F_{2}$ result from the minimization of energies $E_{1}$ and $E_{2}$.

\section{PDE image processing using $\mathrm{CNN}$}

The design of adequate templates is necessary in order to use $\mathrm{CNN}$ for gray-scale image processing. The design is even more difficult if we take the fact that the CNN chips have certain limits into account, namely, single layer processing is supported and linear templates of $3 \times 3$ dimension can be used. The templates for gray-scale images can be designed by making a minimization of the energies or the cost functions [11], but we must frequently take necessary additional conditions for CNN processing into consideration.

The proposed adaptive image enhancement presumes that the attached transfer function depends on the local, or in some cases the regional, intensity level and contrast.

The image processing is based on an iterative algorithm. The adaptive image enhancement presumes also that the number of imposed parameters at the beginning of the algorithm should be as small as possible, and that the characteristics obtained from the initial image are predominant. The image enhancement method proposed in the paper aims at this purpose, as well as the possibility of direct implementation on an existing CNN$\mathrm{UC}$, in a single step, using only linear templates of $3 \times 3$ dimension.

Let us consider the energy function $\mathrm{E}$ defined as follows $[7,16]$ :

$$
\mathrm{E}(\Phi, \mathrm{G})=\underbrace{\iint \mid \nabla \Phi \|^{2} \mathrm{dxdy}}_{\substack{\text { Smoothness } \\
\text { Constraint }}}+\underbrace{\lambda|\mathrm{G}|}_{\begin{array}{c}
\text { Edge } \\
\text { Penalty }
\end{array}}
$$

Minimizing function $\mathrm{E}$ requires estimating two processes, the continuous segmented field, $\Phi$ and a binary edge process, $G$ where $\lambda$ is a scalar parameter; it contains two terms, smoothness constraint and the edge penalty. Thus, the function formalizes a tradeoff between image smoothness (denoising) and edge detection (deblurring).

Aintpol2.tem is resulted based on the constrain conditions presented in paper [11] through the minimization of the energy smoothness term. Aintpol2.tem has only template A:

$$
\mathrm{A}=\begin{array}{|c|c|c|}
\hline 0 & 0.25 & 0 \\
\hline 0.25 & 0 & 0.25 \\
\hline 0 & 0.25 & 0 \\
\hline
\end{array}
$$

In the first approximation, in order to implement the method on a single $\mathrm{CNN}$ layer, the cost function corresponding to the edge penalty term is chosen similarly to the smoothness term with the opposite sign. Template $\mathrm{B}$ and $\mathrm{z}$ result in the same design constrain condition [11]:

$$
\mathrm{B}=\begin{array}{|c|c|c|}
\hline 0 & -1 & 0 \\
\hline-1 & 4 & -1 \\
\hline 0 & -1 & 0 \\
\hline
\end{array} \quad \mathrm{z}=0
$$

The following template (enhan.tem) result from relations $(6,7,8)$ :

$$
\mathrm{A}=\begin{array}{|c|c|c|}
\hline 0 & 0.25 & 0 \\
\hline 0.25 & 0 & 0.25 \\
\hline 0 & 0.25 & 0 \\
\hline
\end{array} \quad \mathrm{B}=\begin{array}{|c|c|c|}
\hline 0 & -\lambda & 0 \\
\hline-\lambda & 4 * \lambda & -\lambda \\
\hline 0 & -\lambda & 0 \\
\hline
\end{array} \quad \mathrm{z}=0
$$

where $\lambda$ is a scalar parameter which defines the ratio between smoothness and contrast enhancement. Smoothness is dominant for values of $\lambda$ lower than one, while for values greater than one, the contrast enhancement predominates. 


\section{Results of testing the adaptive image enhancement}

In this section the experimental results obtained by using the "CadetWin" (CNN Application Development Environment and Toolkit under Windows [17]) are presented. Let us consider an image with low contrast and noise (see Figure 3a).
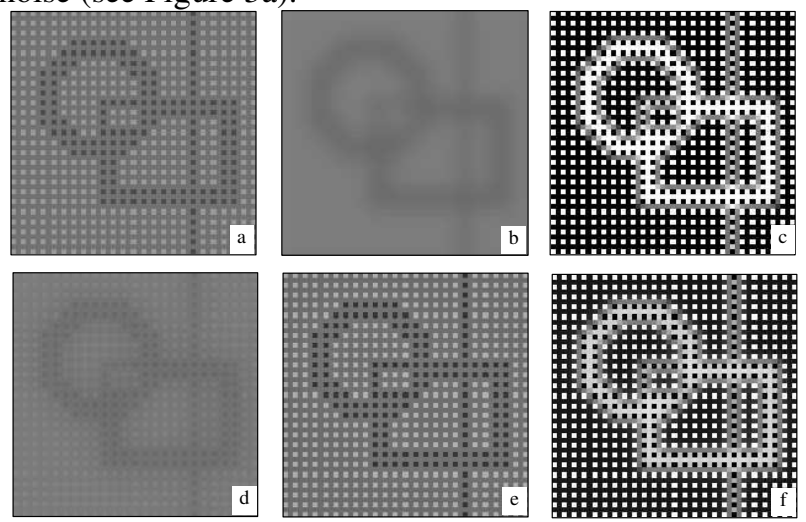

Figure 3: (a) initial image; image resulted by using enhan.tem ((b) with $\lambda=0$; (c) with $\lambda=2$; (d) with $\lambda=0.05$; (e) with $\lambda=0.4$; (f) with $\lambda=1.5$ ).

This image will be processed by using templates that result from (6) for the different values of $\lambda$. If $\lambda=0$ only the averaging of image is produced (Figure $3 \mathrm{~b}$ ), which produces the lost of the edges. The local contrast is maximum if $\lambda=2$, which determines that the pixels can have almost only extreme values +1 or -1 . In Figure $3 \mathrm{c}, \mathrm{d}, \mathrm{e}$ the results of the initial image processing for: $\lambda=0.05, \lambda=0.4$ and $\lambda=1.5$, respectively are presented.

The evaluation of the processing method's performances can also be made if the image, which results from the edge detection, is analyzed (Figure 4).
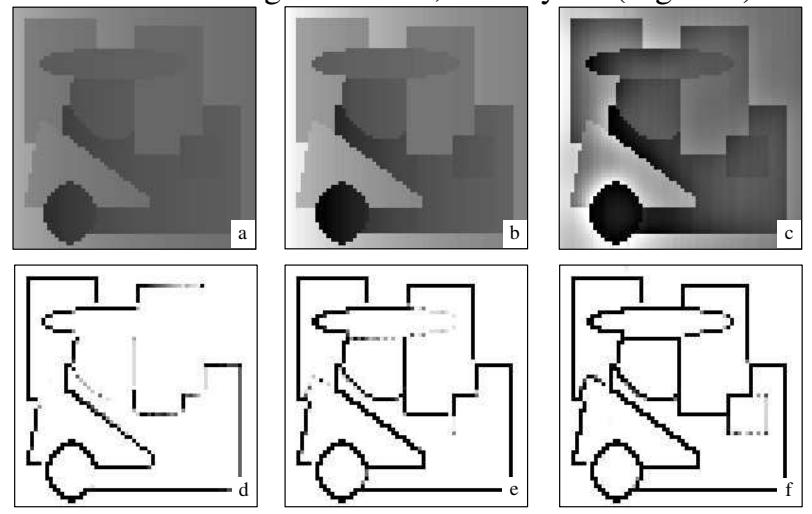

Figure 4: (a) initial image; (b) global scaled image; (c) enhanced image using enhan.tem; (d) edge detection on the initial image; (e) edge detection on the global scaled image; (f) edge detection on the enhanced image.
For edge detection, the same template edgegray.tem was used [17]. It can be observed that the edge detection is satisfying, robust, only for the globally scaled image and for the preprocessed image using enhan.tem

In the case of Computer Tomograph (CT) images enhan.tem can be used for low contrast image enhancement (see Figure 5).
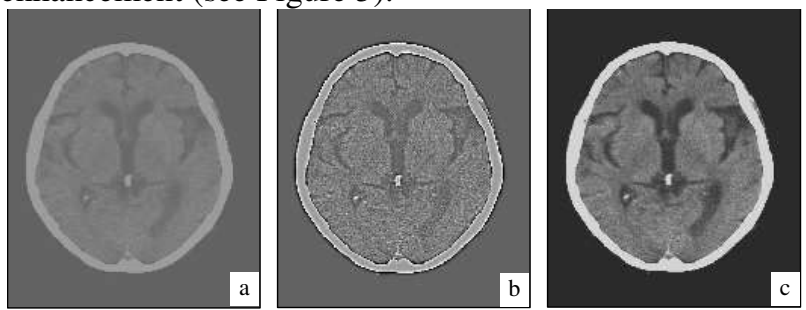

Figure 5: (a) low contrast initial image; (b) enhanced image; (c) global scaled image.

In the case of low contrast cardiac images we can use the same templates. The results are presented in the figure 6 and 7.
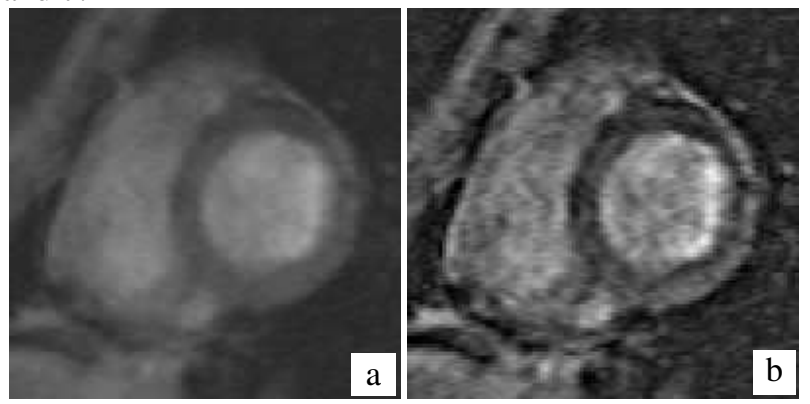

Figure 6: (a) low contrast initial image; (b) enhanced image using enhan.tem.
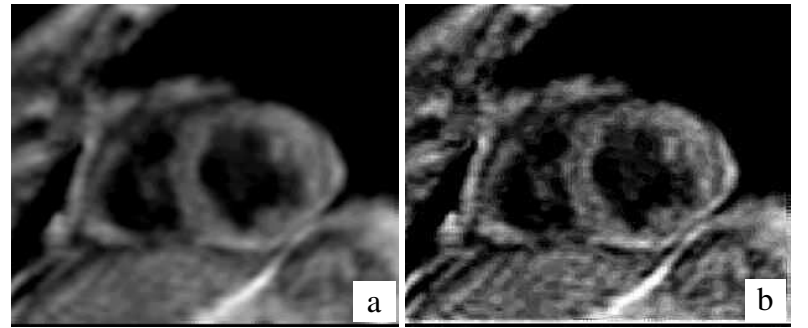

Figure 7: (a) low contrast initial image; (b) enhanced image using enhan.tem. 


\section{Conclusions}

In the paper an medical image enhancement method has been presented, which takes the increase of the contrast into consideration, but also the possibility of direct implementation, on a CNN chip, in a single step. This preprocessing step provides the reduction of computing time, by using complete parallel processing.

There are complex mathematical models with high performances available for image denoising simultaneously with the contrast and contours enhancement [6-10]. Even if day by day a more significant computing power for serial processing is available, for the time being the serial methods are difficult to implement in real time. On the other hand, although CNN parallel processing ensures a computingtime reduction, the sophisticated methods are difficult to implement on a single CNN chip with a single layer, which uses space-invariant linear templates of $3 \times 3$ dimension. This apparent contradiction between the level of the theoretical solutions and the real performances of the CNN computing methods can constitute an eloquent example about how two research fields that are in a permanent competition can be mutually developed.

The CNN chips to be designed and produced will allow the use of CNN methods and other already known PDE image processing methods. The implementation of these computing methods by CNN can be approached by using a similar procedure described in the paper, through the minimization of the combined cost functions for the design of the gray-scale templates.

\section{References}

[1] Chua LO, Yang L. Cellular Neural Networks: Theory and Applications IEEE Transactions on Circuits and Systems 1988; 35:1257-90.

[2] Roska T, Chua LO. The CNN universal machine: an analogic array computer. IEEE Transactions on Circuits and Systems 1993; 40:163-73.

[3] Linan G, Espejo S, Dominguez-Castro R, Roca E, Rodriguez-Vazquez A. CNNUM3: A mixed-signal $64 \times 64$ CNN Universal Chip. Proceedings of International Conference on Microelectronics for Neural, Fuzzy and Bio-inspired Systems 1999; MicroNeuro99:61-8.

[4] Crounse KR, Chua LO. Methods for image processing and pattern formation in cellular neural networks: A tutorial. IEEE Transactions on Circuits and Systems I: Fundamental Theory and Applications 1995; CAS-I-42:583-601.

[5] Rekeczky C, Roska T. Calculating local and global PDEs by analogic diffusion and wave algorithms. Proceedings of the European Conference on Circuit Theory and Design 2001; II: $17-20$.
[6] Casellas J, Morel JM, Sapiro G, Tannenbaum A., Introduction to the special issue on partial differential equations and geometry-driven diffusion in image processing and analysis. IEEE Transactions on Image Processing 1998; 7:269-73.

[7] Perona P, Tartagni T. Diffusion networks for on-chip image contrast normalization. Proceedings of IEEE International Conference on Image Processing 1994; 1-5.

[8] Sapiro G, Casellas V. Histogram modifications via differential equations. Journal of Differential Equations 1997; 135:238-68.

[9] Alvarez L, Guichard F, Lions PL, Morel JM. Axioms and fundamental equations of image processing. Arch. Rational Mechanics 1993; 123:8.

[10] Chen Y, Barcelos CAZ, Mair BA. Smoothing and edge detection by time-varying coupled nonlinear diffusion equations. Computer Vision and Image Understanding 2001; 82:85-100.

[11] Gacsádi A, Szolgay P. Interpolation of 2D signals using CNN. Proceedings of the European Conference on Circuit Theory and Design 2001; I:349-52.

[12] Gonzalez RC, Woods RE. Digital image processing. Addison-Wesley Publishing Company 1993.

[13] Szabó T, Barsi P, Szolgay P. Application of analogic CN algorithms in telemedical neuroradiology. Proceedings of IEEE International Workshop on Cellular Neural Networks and their Applications 2002; 579-86.

[14] Csapodi M, Roska T. Adaptive histogram equalization with cellular neural network. Proceedings of IEEE International Workshop on Cellular Neural Networks and their Applications 1996; 81-6.

[15] Brendel M. Adaptive image sensing and enhancement using cellular neural network universal machine. Research report, Analogical and Neural Computing Laboratory, Computer and Automation Institute, Hungarian Academy of Sciences, Budapest DNS-9-2000.

[16] Mumford D, Shah J. Boundary detection by minimizing functionals. Proceedings of IEEE Conference on Computer Vision and Pattern Recognition 1985; 22-6.

[17] CadetWin CNN application development environment and toolkit under Windows. Version 3.0, Analogical and Neural Computing Laboratory, Hungarian Academy of Sciences, Budapest, 1999.

Address for correspondence:

Name: Cristian GRAVA

Full postal address: 5, Armatei Române Str, 410087 , Oradea, Romania

E-mail address (optional): cgrava@uoradea.ro 